\title{
The Astrosociological Paradigm: The Interplay between Ecologies and Environments
}

\author{
Christopher M. Hearsey ${ }^{1}$ \\ Astrosociology Research Institute, Huntington Beach, California, 92647, USA \\ University of Mississippi School of Law, University, Mississippi, 38677, USA \\ Jim Pass $^{2}$ \\ Astrosociology Research Institute, Huntington Beach, California, 92647, USA
}

\begin{abstract}
This paper discusses, analyzes, and contrasts some of the terminologies used in sociological, ecological, and environmental literatures as they relate to the astrosociological paradigm. Astrosociology is concerned with the two-way relationship between outer space and human society. This relationship includes the vitally important interplay among ecologies and environments on Earth and in outer space. In general, ecology is defined as the relationship between the distribution, abundance, and relations of organisms. On the other hand, environment is defined as an abiotic area, which may or may not be capable of supporting biotas. Within the astrosociological paradigm focusing on contemporary issues and events, we are mainly concerned with humans from the Earth environment maintaining some sort of relationship with various space ecologies, but also mindful of how the space environment surrounding Earth affects terrestrial societies. This paper emphasizes the point that the relationship between ecologies and environments is far more complicated than many realize, having deeper consequences for policy-makers and law-makers, scientists, engineers, and other fields that analyze the utility of outer space. We contend that analyzing ecology and environment as distinctive elements will engender a deeper view of the manifestation of astrosocial phenomena and thereby provide a better framework in which to analyze how such phenomena develop and evolve. Moreover, studying their interactions within an established ecosystem produces even greater insights. This type of approach exposes the essence of astrosociological analysis, and thus it represents a fundamental aspect of the relationship between outer space and humankind.
\end{abstract}

\section{Introduction}

A STROSOCIOLOGY is the study of the two-way relationship between Earth and outer space. Astrosociologists seek to construct research programs that investigate astrosocial phenomena. Astrosocial phenomena arise from the interactions among the Earth and space ecosystems. The focus of astrosociology is to explore how astrosocial phenomena develop and affect human beings, whether on Earth or in outer space. To further this understanding, this paper draws upon the terminology of several fields; namely sociological, ecological, and environmental studies, to make clear the epistemological approach to the study of astrosocial phenomena and its importance to human society.

Space ecologies and environments are often used interchangeably due to a misunderstanding that they are virtually the same thing. However, when viewed from the astrosociological paradigm they are not identical. In fact, the distinction between these two concepts represents a fundamental principle in astrosociology that follows from biotic and abiotic research in the fields of ecological and environmental studies. Moreover, when environment and ecology are viewed together, it represents the dynamic interaction of the biotic and abiotic that we have termed as an "ecosystem." From the astrosociological perspective, the study of ecosystems not only entails the scaling typologies in the traditional ecological studies, but also includes the ontological basis for examining the human connection to outer space. Furthermore, astrosociology is concerned with the interactions between these two ecosystems. Below

1 (1) Deputy Executive Officer, Astrosociology Research Institute, P.O. Box 1129, Huntington Beach, CA, 92647; and (2) J.D. Candidate (2013), University of Mississippi School of Law, University, MS, Student Member.

${ }^{2}$ Chief Executive Officer, Astrosociology Research Institute, P.O. Box 1129, Huntington Beach, CA, 92647, Regular Member. 
we first define the terminology that represents the study of astrosocial phenomena as they pertain to ecosystems. Second, we discuss the three types of interactions that take place between the Earth ecosystem and the space ecosystem. Third, we offer some examples of ecosystems familiar to those who study the varied disciplines that deal with outer space studies, as well as traditional fields of study. Finally, we provide some recommendations for future courses of study.

\section{A. Key Definitions}

\section{Astrosociology and Astrosocial Phenomena}

Astrosocial phenomena represent the fundamental concept of the field of astrosociology. To be precise, astrosociology is defined as the study of astrosocial phenomena (i.e., the social, cultural, and behavioral patterns related to outer space). ${ }^{1}$ This definition ties humanity to outer space in a fundamental way. For example, astronomical phenomena do not involve human beings, such as when a star goes supernova. On the other hand, individuals witnessing the star explode through a telescope may be characterized as an astrosocial phenomenon because there is a human connection of some kind involved. Equally, astrosocial phenomena can arise also from the social effects following a close flyby of an asteroid. Hence, how the space ecosystem and the Earth ecosystem interact and affect humans at every level of social construction is the principle investigative focus of the field of astrosociology.

Expanding on the concept of astrosocial phenomena will provide a more comprehensive understanding of the astrosociological paradigm. This will prepare the reader with the knowledge required to follow the remainder of the thesis presented below. We attempt to show that the field of astrosociology becomes necessary to comprehend the pertinent issues involved when studying both (1) human expansion into space and (2) the increasing influence of space on terrestrial societies. The types of astrosocial phenomena that arise can help determine the forces that shape decision-making, evolutionary processes, and the future of our species.

\section{Environment}

We define environment as an abiotic area, which may or may not be capable of supporting biotas. This definition follows from the varied typologies defined in ecological and environmental literatures. Generally, an environment consists of a physical place without reference to biota, which on Earth can range from rock formations to a building. In outer space, examples of environments range from the artificial (or engineered) types that are constructed by humans, e.g., space stations, launch vehicles, etc., to the natural types that exist without human intervention, e.g., surface of a planetary body. On Earth, an analogy of an artificial environment is a climatecontrolled building, especially in a harsh environment such as Dubai in the middle of summer. Arctic bases provide researchers with even better analogs.

Natural environments - and thus their ecosystems - are rarely totally isolated (perhaps deep caves), but artificial environments are designed with isolation in mind to protect inhabitants from the harsh conditions of the space environment in which their ecosystem exists. Thus, the adaptations that communities must make in response to their environment characterize the astrosocial phenomena that develop in the resulting ecosystem. That is, astrosocial phenomena become the products of adaptations of communities and individuals to their surrounding conditions. Human beings must largely adapt to natural environments. Conversely, human beings can construct artificial environments to suit their requirements. Of course, artificial environments are difficult to construct, especially on a large scale, but they provide potential advantages for not only survivability, but livability as well.

As Pass has noted, space environments refer to:

the idea of multiple locations, each with its own unique mix of physical characteristics found in specific regions of space...Each environment possesses the potential of becoming an [ecosystem] if humans ever visit there. Each [environment] possesses its own set of conditions that require specific adaptations by humans to varying conditions. ${ }^{2}$

Thus, it is vital to keep in mind that each locality in space has its own unique conditions. Astrosociology does not assume that humans will adapt to every environment in the same way, or at all. If one introduces human beings to particular environments, each would influence social behavior, biological health, and genetic constitution in unique ways.

In addition, the scale of environments can vary and affect ecologies, and in turn affect ecosystems, in a variety of ways. First, we note that Earth and outer space are separate units of analysis. Second, each environment may be scaled beyond the metaphysical intersection between the Earth and outer space environments. Some Earth environments are just as varied and inhospitable as outer space environments. Nevertheless, the ontological basis of astrosociology views Earth and outer space as dichotomous units in terms of how each affects humans individually or separately. Moreover, without the presence of intelligent life in either environment, it would be useless to discuss astrosociology. Fourth, scaling can affect how humans are affected by astrosocial phenomena. For example, the 
impact of a space object onto the Earth's surface will give rise to different responses depending on whether a person or society is on Earth, above the Earth in a space station, or on another planetary body. Thus, scale of the environment can affect the impact and nature of astrosocial phenomena.

\section{Ecology}

We define ecology to mean the relationships between the distribution, abundance, and relations of organisms. ${ }^{3}$ Hearsey gave a preliminary outline of this definition noting that "outer space ecology as the relationship between the distribution, abundance, and relations of organisms within the outer space environment." ${ }^{44}$ However, the definition of ecology is broader depending upon the types of biotas under observation. As noted above, the metaphysical distinction of Earth and outer space as interacting units only makes sense when we consider the biotic relationships among and between humans in different social constructions. Nonetheless, humans represent the main unit of analysis under astrosociology, but the biotic relationships humans have with other organisms may be varied depending upon the environment. In addition, an organism could possibly predominate in its relationship with humans. For example, organisms mutated in outer space or from an extraterrestrial source could have inert, positive, or negative effects on human biology.

Our definition of ecology is devoid of the environmental element in order to distinguish between the biotic and abiotic units of analysis. However, scholars use the term ecology interchangeably with the term "ecosystem" as well as in co-reference to biotas in an environment. ${ }^{5,6}$ Furthermore, the general focus of ecological research relates to the study of ecosystems and, to some degree, the research takes a normative position relative to particular theses. ${ }^{7}$ Some ecological literature focuses on the dynamics of life in defined environments, and some literature argues for prescriptive solutions to protect or preserve nature, as well as to promote ethics for the cohabitation and/or coevolution with other life forms. ${ }^{8,9}$ However, astrosociology does not attempt to take a normative position or promulgate one ethic over another. Instead, astrosociologists concern themselves with how astrosocial phenomena arise within ecosystems, as well as how they affect the creation and evolution of human social structures.

There exist different types of ecologies both in typology, but also within certain fields. We may define the term space ecology as the study of relationships between the distribution, abundance, and relations of organisms with a particular space environment. In contrast, human ecology, as developed within the so-called Chicago School of Sociology that focused on urban sociology, originally referred to the sociological study of the spatial and temporal interrelationships between humans and their economic, social, political organization within a defined environment, whether this environment is well defined or more nebulous. ${ }^{10}$ Later, other social scientists and especially sociobiologists added biological factors to the traditional sociological approach developed by Robert Park and others at the University of Chicago in the 1920s. ${ }^{11}$ Environmental sociology, on the other hand, tends to view society and culture as more important than biological influences within a specific environment. ${ }^{12}$ While they still focus on human beings, these researchers and scholars downplay the biological influences to a significant extent. In the case of space environments, one may expect that their impact on human biology becomes a greater concern than exists in terrestrial environments.

The authors take the stance that the original position taken by the modified version of human ecology, which adds biological factors, is the most appropriate model for humans living in space environments, as social and biological effects exist in space environments. On the other hand, the authors realize that biological determinism alone is unable to explain all forms of astrosocial phenomena. However, we have adapted the term ecosystem to represent the fusion between the biotic (ecology) and abiotic (environment). Below we discuss the meaning of ecosystem under the astrosociological paradigm.

\section{Ecosystem}

An ecosystem refers to a complex community of organisms that exist within a physical place or environment that interacts with its environment and functions as a distinct ecological unit. The authors assume that this concept includes all organisms in a particular environment, and thus includes humans as well as other organisms. On Earth, the boundaries of a given ecosystem often are quite nebulous. Sometimes, however, ecosystems have well defined boundaries such as the organization of different species within a lake. In space environments, the latter model is overwhelmingly common because human hands and machines (e.g., a spacecraft, space station, or space habitat on the surface on a cosmic body) construct the boundaries involved. Of course, these types of environments exist for the protection of humans and other life forms. They exist to protect them from the many harmful conditions that comprise the natural space environment. On Earth, the environments that exist are much less hostile, and protection normally consists of coping with the weather and other outside elements. Nevertheless, environmental conditions on Earth can become life threatening, and thus researchers can learn lessons from how humans cope with dangerous situations of various types. 


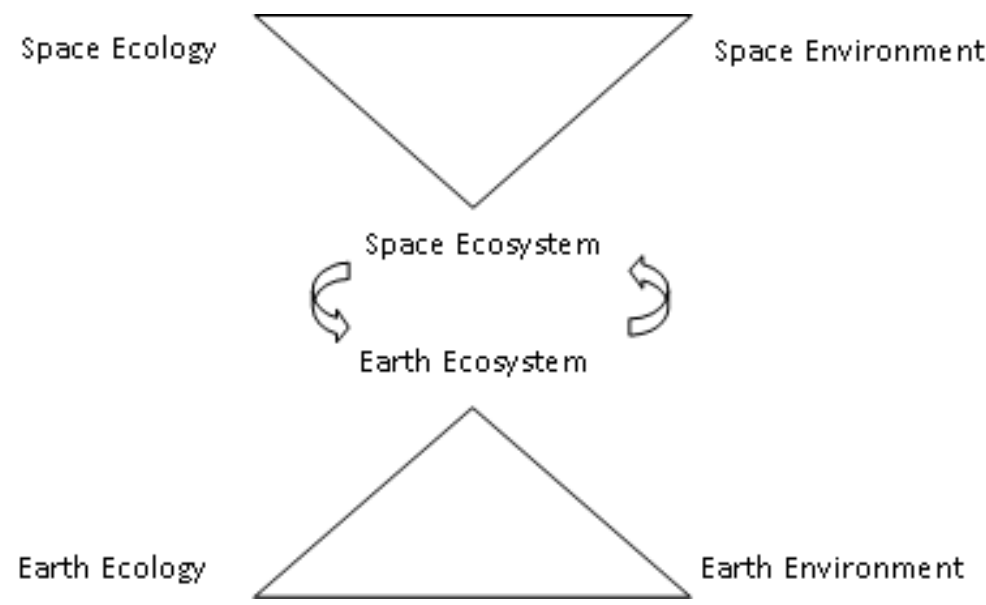

Figure 1 - Diagram of how the elements of ecology and environment are connected as interacting metaphysical units as a basis for the investigation of astrosocial phenomena.

Figure 1 above illustrates how environments and ecologies function together and represents the metaphysical connection between the space and Earth ecosystems. The top triangle represents the connection between the space ecology and the space environment, as well as how they interact to form the space ecosystem as a unit of analysis. The bottom triangle represents the connection between the Earth ecology and the Earth environment, as well as how they interact to form the Earth ecosystem as a unit of analysis. The interactions that occur between the space ecosystem and the Earth ecosystem are depicted between the two triangles. The dynamics between the two elements occur in three forms depicted in figure 2. However, the effects of these interactions pass through to affect the environments and ecologies individually and collectively on Earth or in outer space depending on the type of event being investigated.

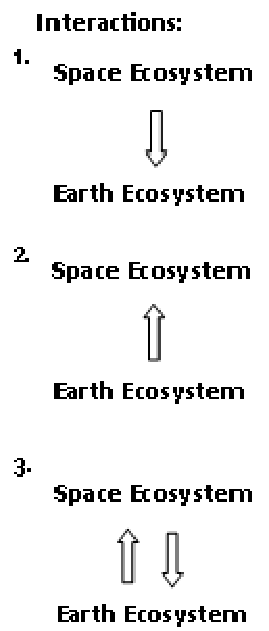

Figure 2 - Interactions between the Space and Earth Ecosystems

Figure 2 above represents the three types of interactions that occur as vectors between the space ecosystem and the Earth ecosystem. The first interaction occurs as a one-way vector between the space ecosystem and the Earth ecosystem. In this interaction, the space ecosystem directly affects the Earth ecosystem. For example, an astronomical event observed or has the potential to affect Earth indirectly can produce astrosocial phenomena on Earth as long as human beings are aware of them.

The second interaction occurs as a one-way vector between the Earth ecosystem and the space ecosystem. In this interaction, the Earth ecosystem directly affects the space ecosystem. For example, the launch of satellites into 
Earth orbit for telecommunications enables the Earth ecosystem to affect space in at least two distinct ways. First, the presence of satellites in orbit takes up physical space and thus causes congestion. Second, the use of satellites to enable data transmission treats space as a tool for human activities on Earth.

The third interaction occurs as a reciprocal two-way vector between the space ecosystem and the Earth ecosystem. Here, the two-way interaction between the space and Earth ecosystems generates the greatest set of investigations. Because the interaction between humans and outer space has occurred and continues to occur, the effects that perpetuate and evolve from the interactions continue to persist. The movement of humans and material from Earth into outer space can initiate certain types of astrosocial phenomena. Conversely, the presence of humans and material in space will perpetuate interactions in the space ecosystem that will affect how humans or any biotas interact in outer space and on Earth.

\section{The Role of Ecosystems in Astrosociology}

This paper has two major themes. The first is that space environments offer space ecologies with unique conditions and challenges that affect human social organization and social life on a multitude of different levels. Additionally, the fact that space environments may differ drastically from one another means that space societies will organize themselves in drastically different ways. Adaptation strategies will differ from location to location and will involve drastic steps compared to Earth environments in order to make them both survivable and livable. Members on long and/or distant expeditions will find that many of the things taken for granted on Earth must be manufactured or sacrificed in space environments.

Secondly, one cannot understand human behavior in space properly without taking into consideration the influences of the environment. It is vital to study the entire human ecosystem in a particular locale in space, just as it is on Earth. One may compare the dangers to human health and survival inherent in space environments, both predictable and unforeseen, to the harshest climates on Earth if they had no breathable atmosphere or magnetic field to protect life, though Earth analogs are far less hazardous in comparison. Nevertheless, Earth analogs can provide important lessons for organizing social life in hostile environments. Knowledge of any type that can help prepare humanity for adapting to unimaginably harsh conditions will make the transition that much more successful.

\section{A. Human Ecosystem / Space Ecosystem}

An important focus of astrosociology is on the concept of a human ecosystem, defined here as the interaction between a human social group and its surrounding environment. This type of ecosystem involves humans interacting with one another and with their environment to form an ecological unit. While other organisms may exist in terrestrial ecosystems and introduced into extraterrestrial ecosystems, the focus of study in on human beings interacting with each other and elements of their physical environments (both artificial and natural). Human ecosystems vary in complexity from a small band or group at one end of the continuum to societies and planetary ecosystems at the other. For example, a human ecosystem in space, referred to as a "space ecosystem," must overcome extremely harsh environmental conditions, to be sure, so it is imperative that astrosociologists have access to how astrosocial phenomena manifest themselves and how they influence social interaction.

\section{B. Space Society}

When considering a human ecosystem, one must take into account the structure of the social system in which humans live. The environment partly shapes the ecosystem in which it resides although the requirement to create social structures that regulate human behavior and meet the needs of the people also shapes the ecosystem. Additionally, conditions can change to produce new challenges to which social systems must adapt, and such adaptations can result in adjustments to the organization of a particular space society. The concept of space society is therefore important to consider, which is defined as the following:

a space colony/settlement in which members of the population (1) share a common culture, (2) live within a closed physical environment, and (3) cooperate with one another, social groups, and institutions in order to meet the social needs of all its citizens. $^{13}$

The space society resides in the physical environment and thus the society is part of the human ecosystem. People construct the social environment, but they must adapt to the space environment. On one level, the natural physical environment shapes the design and attributes of the space habitat, which is the artificial environment. For example, humans who decide to live in a natural physical environment characterized by a high level of radiation must devote much of their construction costs to building a habitat that protects inhabitants. It may even force them to place the habitat in a crater or wall of a hill or valley. These characteristics also affect their social system in a variety of ways. When the society grows large enough to construct social institutions, problems with coping with the radiation may 
force political attention to address them and they may need to redirect economic resources toward mitigating them even further.

As just alluded to above, space societies can exist in various sizes, and greater population sizes correspond to greater complexity and impersonalization, ${ }^{14}$ which includes micro-societies, mini-societies, and "full-fledged" societies. The human ecosystem first created when a social group settles in a specific area on Mars, for example, will tend to be small. Therefore, the physical environment will place extreme pressures on the micro-society to survive. Long-term habitability would require a growth in population size so that adaptation to the environment is less intense on each person.

\section{Broadening the Earth-Centric View}

The Earth-centric view is not an inaccurate perspective; it is just incomplete. Before humans could venture into space, it was quite natural for people to concern themselves with terrestrial matters. While they could dream about going into space, they could not actually accomplish the feat. Gaining a space-centric view would require escaping the confines of Earth's gravity well.

When finally accomplishing this feat in 1961, a new competing perspective began to emerge to challenge previous notions of where human beings fit into the larger scope of things. Human beings could finally see the Earth as a true planet for the first time. The environmental movement received a boost when people saw the Earth from space, especially when the entire planet was viewed from photographs taken by astronauts on the Moon. Thus, we believe that to communicate astrosociology as a multidisciplinary field, astrosociologists should be aware of nexus between terminologies. Taking a wider view of the connection between outer space and humanity will enable the astrosociological paradigm to become clearer. Astrosociology does not seek to compete with other disciplines, but to enhance our understanding of the world and how humanity fits within various ontologies.

\section{Essential to Astrosociology: The Two-Way Relationship}

There is a two-way relationship between space and society. Many who first learn of astrosociology assume that the field only focuses on humans living and working in space. This is incorrect as this entire discussion implies. The space environment affects life on Earth as well. The key to determining astrosocial phenomena is to investigate the connection between humanity and outer space. Therefore, humans living on Earth can be affected by the surrounding space environment (e.g., the Earth hit by harmful solar flares), from humans working in space (e.g., astronauts conducting experiments), or from space assets manufactured by humans (e.g., receiving data from weather satellites). The point is that space already affects human lives and the space environment surrounding Earth is a crucial thing to understand because it can impact negatively on the planet's surface and therefore its biosphere.

This two-way relationship implies that the dawn of the space age initiated a new reality in which life on Earth became linked to space in a significant way that has only increased in impact since it began. Even today, many people overlook the influence of astrosocial phenomena on their daily lives. Recognition of their connections to the cosmos would result in new ways of viewing social reality and therefore providing them with the potential to expand their existing assessments of future possibilities. In many ways, accepting the idea that outer space is actually part of their environment at larger scales than they are used to opens up new possibilities about future progress.

\section{The Overview Effect}

Humans' perceptions of their surroundings determine much of their behavior. Before humankind reached Earth orbit, the view of social life was limited to a grounded perspective as it related to the environments that could involve human interaction. Once achieving Earth orbit, humans suddenly interacted with one another beyond the confines of Earth's surface or other terrestrial environments.

As White has noted, the Overview Effect involves how space alters our perceptions of Earth and our relationship to it. The interesting thing about the Overview Effect is that is illustrates how space can affect human perception, which in turn affects human behavior. Based mostly on anecdotes from astronauts and to a much lesser extent on those of others who have viewed the photographs of the Earth as a single orb floating in space, the experience itself makes one more cognizant of certain facts such as the need to protect the environment, the realization that planetary peace is possible or at least desirable (and thus racism and sexism are counterproductive as is fighting among ourselves at various levels), the possibility of a one world government, and humanity's connection to the cosmos. ${ }^{15}$ Those with religious backgrounds see the work of God involved while those without such backgrounds view it as some form of enlightenment that they gain from the experience of seeing Earth as a true planet for the first time. The Overview Effect involves the idea that human space exploration can open the way for new possibilities in human evolution, and this may result in new types of ecosystems and space societies.

How could the Overview Effect play out in other space environments? Perhaps it will encourage space explorers beyond Earth orbit to place much of their planning toward a functional space society beyond just ensuring that the 
human beings are safe from dangerous conditions in their surrounding environment. In some ways, these explorers and settlers will appreciate their connection to the cosmos more strongly and thus perhaps they will incorporate a greater number of metaphysical elements into their ecosystem beyond just the practical elements related to survival. It is quite conceivable that different space societies will evolve very differently due not only to adaptations to their physical environments but also based on how they perceive their existential relationships to these environments. The application of social meaning to their lives in space environments will prove extremely important in terms of what types of ecosystems they develop.

\section{Conclusion}

This paper represents an exploratory theoretical treatment of the relationships among environments, ecologies, and ecosystems. Therefore, more effort is needed to continue the work that has just begun. The recommendations below provide several avenues in which to expand the effort provided here.

Space environments and the ecosystems that develop in their wake will undoubtedly become a vital area of astrosociological research. In fact, the space environment will play a big role in shaping how ecosystems form and develop, and thus it will also affect human behavior, culture, and a variety of other astrosocial patterns. The astrosociological study of environments and ecosystems, or perhaps termed as space ecology, will serve as a fundamental area of research.

In the end, sustainable survivability within the space environment is the most important factor. However, if one assumes that this state of affairs exists, then one must focus on the interactive effects between the human group and its environment. In turn, it is important to understand how these types of interactions affect individual human behavior, that of components of the space society such as its culture and social institutions, and the organization of the entire society.

\section{Recommendations for Future Research}

This paper has focused mainly on the theoretical aspects of space environments and ecosystems as a starting point for astrosociologists and related scientists/scholars to pursue. Many research questions have come out of this exercise. It is appropriate, then, to list them formally for the convenience of the reader.

Specific recommendations include:

1) incorporation of ecosystem analysis into astrosociological models;

2) apply chaos theory to astrosociological models in order to take into account the inherent instabilities in ecosystems and social systems;

3) construct an investigation into particular astrosocial phenomena using the epistemological approach developed above; and

4) investigate harsh terrestrial environments in which human ecosystems have had to shape themselves based on surrounding conditions.

In conclusion, more research in this area is needed from astrosociologists from a variety of perspectives. Though the theoretical model requires further development, we believe that empirical research must begin on a complementary track. We have enough examples to test on Earth and in low Earth orbit.

\section{References}

\footnotetext{
${ }^{1}$ Pass, J., "Target Viewpoint: Examining the Definition of Astrosociology," Astropolitics, Vol. 9, 2011.

2 Pass, J., "Target Viewpoint: Examining the Definition of Astrosociology," Astropolitics, Vol. 9, 2011, p. 26.

${ }^{3}$ Hearsey, C., "The Nexus between Law and Astrosociology," Astropolitics, Vol. 9, ftn. 15, 2011, p. 31.

${ }^{4}$ Hearsey, C., "The Nexus between Law and Astrosociology," Astropolitics, Vol. 9, 2011, p. 31.

${ }^{5}$ E.g., Loreau, M., et al, "Meta-Ecosystems: a Theoretical Framework for a Spatial Ecosystem Ecology," Ecological Letters, Vol. 6, 2003, pp. 673-679.

${ }^{6}$ E.g., Hobbs, R.J., et al., "Intervention Ecology: Applying Ecological Science in the Twenty-First Century," BioScience, Vol. 61, No. 6, 2011, pp. 442-450.

7 E.g., Dacko, M., and Dacko, A., "Management of the Natural Environment - A Systemic Approach," Polish Journal of Environmental Studies, Vol. 18, No. 2, 2009, pp. 145-150.

${ }^{8}$ E.g., Cairns, Jr., J., "Sustainable Co-Evolution," International Journal of Sustainable Development and World Ecology, Vol. 17, 2007, pp. 103-108.

9 E.g., Haluza-DeLay, R., and Davidson, D. J., “The Environment and a Globalizing Sociology,” Canadian Journal of Sociology, Vol. 33, No. 3, 2008, pp. 631-656.
} 
${ }^{10}$ Park, R. E., and Burgess, E. W. S. (eds.), Introduction to the Science of Sociology, University of Chicago Press, Chicago, 1921.

${ }^{11}$ E.g., Wilson, E. O., "What is Sociobiology," Society, Vol. 6, No. 6, 1978, pp. 10-14.

${ }^{12}$ Dunlap, R. E., and Michelson, W. (eds.),.Handbook of Environmental Sociology, Greenwood Press, Westport, Ct: 2002.

13 Pass, J., "The Astrosociology of Space Colonies: Or the Social Construction of Societies in Space," Space Technology and Applications International Forum (STAIF) Conference Proceedings, 813(1): 1153-1161, reproduced by permission by the American Institute of Physics at the Astrosociology Research Institute (ARI) Virtual Library [online archive],

http://www.astrosociology.org/Library/PDF/Submissons/STAIF_Astrosociology\%20of\%20Space\%20ColoniesPDF.pdf, 2006, accessed August 9, 2010, p. 4.

${ }^{14}$ Pass, J., "The Astrosociology of Space Colonies: Or the Social Construction of Societies in Space," Space Technology and Applications International Forum (STAIF) Conference Proceedings, 813(1): 1153-1161, reproduced by permission by the American Institute of Physics at the Astrosociology Research Institute (ARI) Virtual Library [online archive], http://www.astrosociology.org/Library/PDF/Submissons/STAIF_Astrosociology\%20of\%20Space\%20ColoniesPDF.pdf, 2006, accessed August 9, 2010.

${ }^{15}$ White, F., The Overview Effect: Space Exploration and Human Evolution (2nd ed.), AIAA, Reston, VA, 1998. 